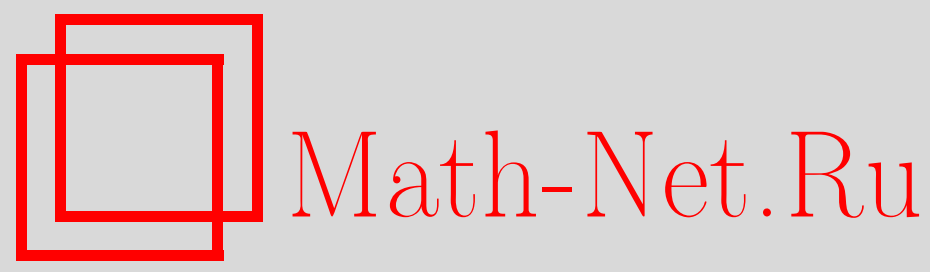

С. Р. Беджанова, Схемы для дизъюнкции, допускающие короткие единичные диагностические тесты, Дискрет. матем., 2010, том 22, выпуск 4, 43-54

DOI: https://doi.org/10.4213/dm1118

Использование Общероссийского математического портала Math-Net.Ru подразумевает, что вы прочитали и согласны с пользовательским соглашением http://www . mathnet.ru/rus/agreement

Параметры загрузки:

IP : 3.80 .181 .102

26 апреля 2023 г., 08:08:54 


\title{
Схемы для дизъюнкции, допускающие короткие единичные диагностические тесты
}

\author{
(c) 2010 г. С. Р. Беджанова
}

\begin{abstract}
В работе исследуются тесты для схем, реализующих дизъюнкцию. В схеме допускается инверсная неисправность на выходе ровно одного из функциональных элементов. Для любого функционально полного базиса $B$ установлено, что при любом $n \geqslant 3$ дизъюнкцию $n$ переменных можно реализовать схемой над $B$, допускающей единичный диагностический тест не более чем из двух наборов. Доказано, что в общем случае этот результат неулучшаем.

Работа выполнена при поддержке Российского фонда фундаментальных исследований, проект 08-01-00863, и программы фундаментальных исследований РАН «Алгебраические и комбинаторные методы математической кибернетики и информационные системы нового поколения».
\end{abstract}

Рассматриваются схемы из функциональных элементов в произвольном полном конечном базисе $[1,2]$, реализующие дизъюнкцию $n$ переменных, где $n \geqslant 3$. Пусть в схемах в неисправное состояние может перейти выход ровно одного из элементов, и это инверсная неисправность. Таким образом, если в исправном состоянии элемент реализует функцию $\varphi$, то при его поломке на выходе элемента реализуется функция $\bar{\varphi}$. Среди всех схем, реализующих $f(\tilde{x})=x_{1} \vee \ldots \vee x_{n}$, будем выделять те, которые допускают тесты минимальной возможной длины.

Функция, реализуемая на выходе схемы при наличии в схеме неисправного элемента, называется функцией неисправности. Пусть $g_{1}, \ldots, g_{k}-$ все возможные функции неисправности схемы $S$, отличные от функции $f$. Множество $T$ наборов длины $n$ называется единичным диагностическим тестом схемы $S$, если для любой пары функций из множества $\left\{g_{1}, \ldots, g_{k}, f\right\}$ в нем найдется набор, на котором эти функции различаются. Через $D(T)$ обозначим длину этого теста, то есть число наборов в нем. Пусть $D(S)=\min D(T)$, где минимум берется по всем единичным тестам для схемы $S$, а $D_{\text {Едт }}\left(x_{1} \vee \ldots \vee x_{n}\right)=\min D(S)$, где минимум берется по всем схемам, реализующим $f(\tilde{x})=x_{1} \vee \ldots \vee x_{n}$. Заметим, что в данной работе при исследовании единичных тестов рассматриваются, как обычно, неизбыточные схемы (определения см., например, [3, 4]).

Теорема 1. Функция $f(\tilde{x})=x_{1} \vee \ldots \vee x_{n}$ может быть реализована неизбыточной схемой, допускающей единичный диагностический тест, состоящий не более, чем из двух наборов.

Доказательство. При конструктивном доказательстве теоремы воспользуемся классификацией базисов из [5]. 


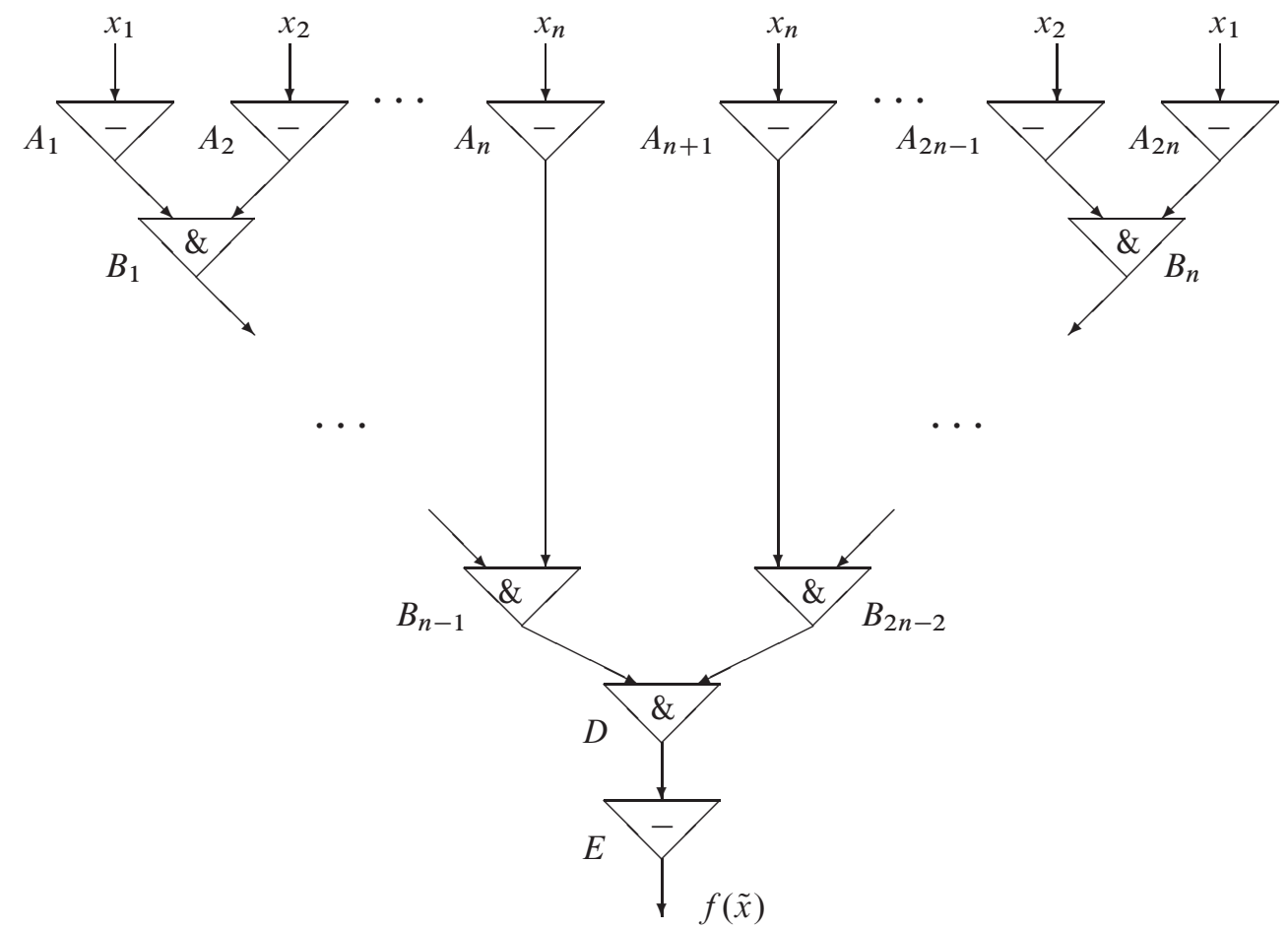

Pис. 1.

1. Базисы $\{x \vee y, \bar{x}\},\{x \vee \bar{y}, \bar{x}\}$. Для таких базисов утверждение теоремы доказано в [6].

2. Базис $\{x \& y, \bar{x}\}$. Функцию $f(\tilde{x})=x_{1} \vee \ldots \vee x_{n}$ в данном базисе можно реализовать схемой, представленной на рис. 1. При переходе в неисправное состояние любого одного из элементов $A_{1}, \ldots, A_{2 n}, B_{1}, \ldots, B_{2 n-2}$ получим функцию неисправности $g_{1} \equiv 1$; при неисправности элемента $D$ или элемента $E$ получим функцию неисправности $g_{2}=\bar{f}$.

Итак, получаем всего две различные функции неисправности, следовательно, $D_{\text {Едт }} \leqslant 2$ (здесь и далее в подобных случаях достаточно воспользоваться определением единичного диагностического теста и теоремой 7 из [3]).

3. Базис $\{\bar{x} \& \bar{y}\}$. Реализуем дизъюнкцию $n$ переменных схемой, представленной на рис. 2 ; в этой схеме подсхема $S_{2}$ устроена точно таким же образом, как и $S_{1}$. Без ограничения общности предполагаем, что в блоке $S_{2}$ отсутствуют неисправности. Если неисправен элемент $A_{1}$ или элемент $B_{1}$, то получаем функцию неисправности

$$
\overline{x_{1} \vee x_{2}} \vee x_{3} \ldots \vee x_{n} \vee f \equiv 1 \text {. }
$$

При неисправности одного из элементов $A_{i}, B_{i}, i \in\{2, \ldots, n-1\}$, получаем функцию неисправности

$$
\overline{\left(x_{1} \vee \ldots \vee x_{i}\right)} \& \bar{x}_{i+1} \vee x_{i+2} \vee \ldots \vee x_{n} \vee f \equiv 1 .
$$

При неисправности $E$ или $E^{\prime}$ на выходе схемы получим $\bar{f}$. В итоге получаем две нетривиальные функции неисправности $g_{1} \equiv 1$ и $g_{2}=\vec{f}$, а это означает, что $D_{\text {Едт }} \leqslant 2$. 


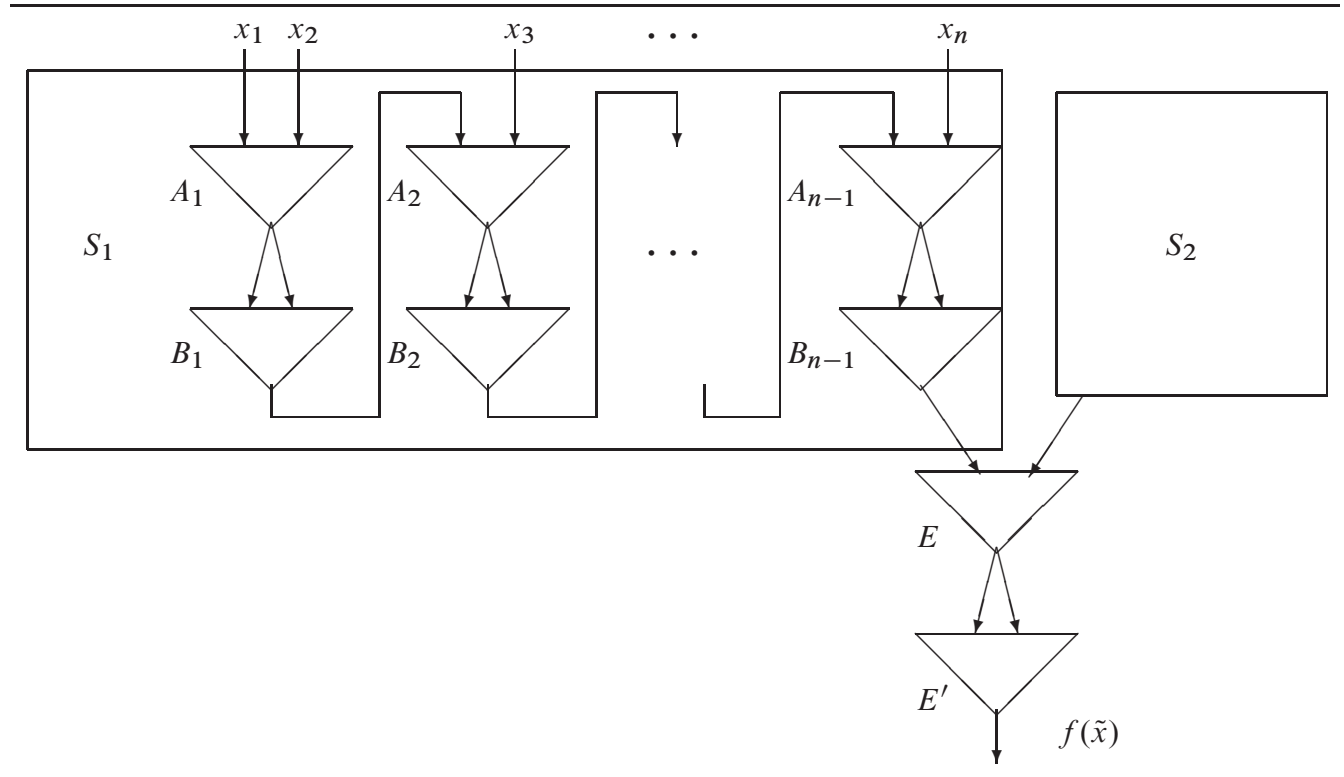

Рис. 2.

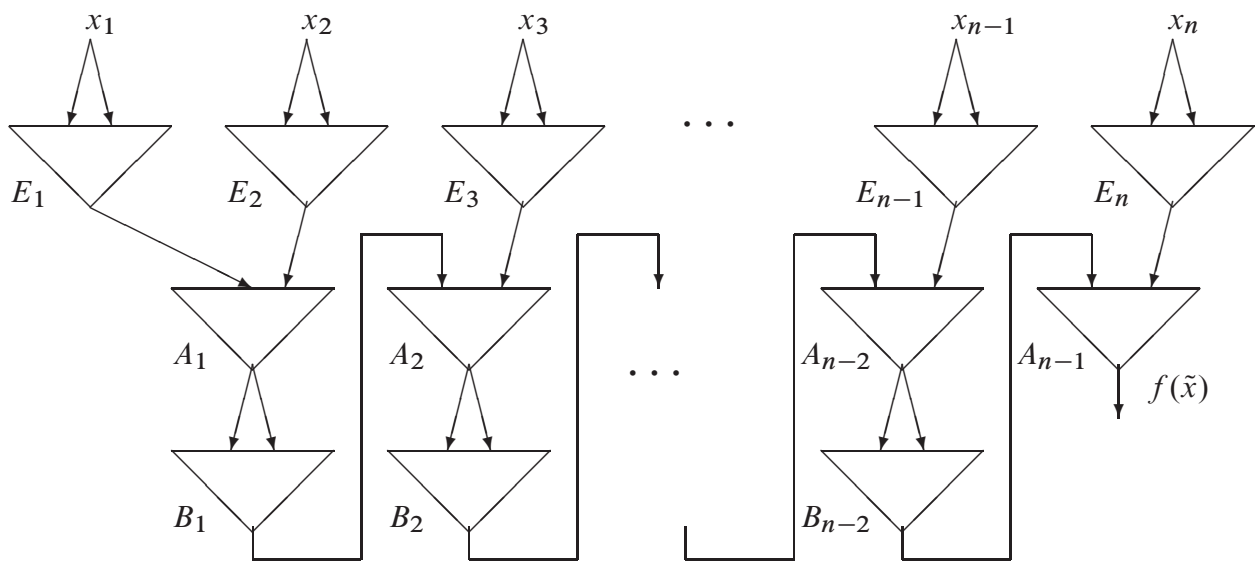

Рис. 3.

4. Базис $\{\bar{x} \vee \bar{y}\}$. Схема $S^{\prime}$ на рис. 3 реализует функцию $f(\tilde{x})=x_{1} \vee \ldots \vee x_{n}$. Эта схема реализует также, как нетрудно заметить, следующие функции неисправности:

$$
g_{i}=x_{1} \vee \ldots \vee x_{i-1} \vee \bar{x}_{i} \vee x_{i+1} \vee \ldots \vee x_{n}
$$

при неисправности элемента $E_{i}, i=1, \ldots, n$;

$$
h_{i}=\bar{x}_{1} \& \ldots \& \bar{x}_{i+1} \vee x_{i+2} \vee \ldots \vee x_{n}
$$

при неисправности одного из элементов $A_{i}$ или $B_{i}, i=1, \ldots, n-2 ;$ и $\bar{f}$ при неисправности элемента $A_{n-1}$. 


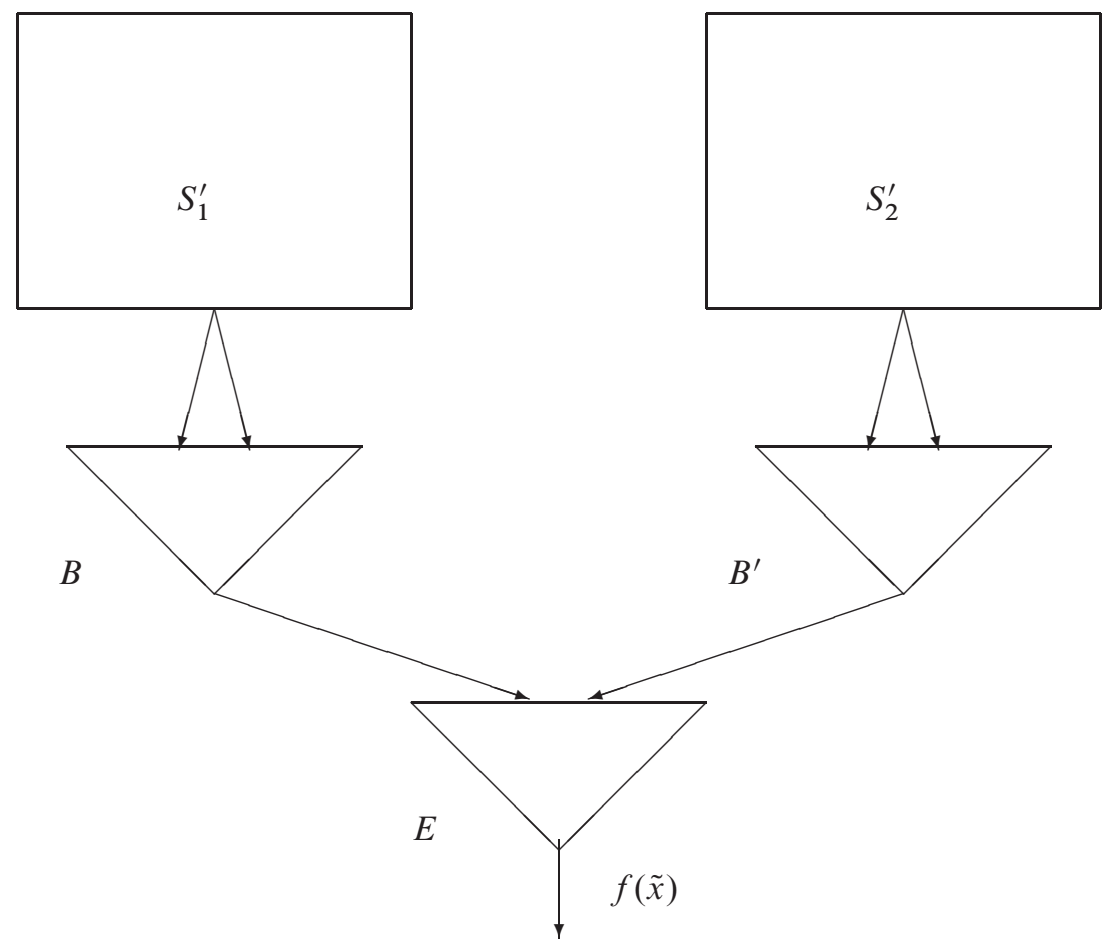

Рис. 4.

Рассмотрим представленную на рис. 4 схему $S$, которая содержит две одинаковые подсхемы $S^{\prime}$ (на рисунке это подсхемы $S_{1}^{\prime}$ и $S_{2}^{\prime}$ ) и реализует функцию $f(\tilde{x})$. Пусть без ограничения общности все элементы из $S_{2}^{\prime}$ и элемент $B^{\prime}$ исправны. Тогда при наличии неисправностей в $S_{1}^{\prime}$ на выходе схемы $S$ получим следующие функции неисправности:

$$
\begin{aligned}
g_{i} \vee f \equiv 1, & i=1, \ldots, n ; \\
h_{i} \vee f \equiv 1, & i=1, \ldots, n-2 ; \\
f \vee \bar{f} & \equiv 1 .
\end{aligned}
$$

При неисправности элемента $B$ получим функцию неисправности $f \vee \bar{f} \equiv 1$, а при неисправности $E$ получим $\bar{f}$. Всего для схемы $S$ возможны две нетривиальные функции неисправности и поэтому $D_{\text {Едт }} \leqslant 2$.

5. Базис $\{x \& \bar{y}, \bar{x}\}$. Реализуем $f(\tilde{x})$ схемой $S$, представленной на рис. 5 , где $S_{1}^{\prime}$ устроена также, как и $S_{1}$ (одновходовые элементы реализуют $\bar{x}$, а двухвходовые реализуют функцию $x \& \bar{y}$ ). На выходах подсхем $S_{1}$ и $S_{1}^{\prime}$ (при наличии в них единственного неисправного элемента) могут оказаться реализованными следующие функции:

$$
g_{1}=\overline{x_{1} \bar{x}_{2} \ldots \bar{x}_{n}}, \quad g_{2}=\overline{\overline{\bar{x}_{1} \bar{x}_{2}} \bar{x}_{3} \ldots \bar{x}_{n}}, \quad \ldots, \quad g_{n}=\overline{\overline{\bar{x}_{1} \bar{x}_{2} \ldots \bar{x}_{n}}}=\bar{f}
$$

На выходе всей схемы возможны следующие функции неисправности: $h_{i}=\overline{\bar{g}} \overline{\bar{f}} \equiv 1$ при неисправности какого-то одного элемента в $S_{1}$ или в $S_{1}^{\prime}, i=1, \ldots, n ; h_{n+1}=\overline{f \& \bar{f}} \equiv 1$ 


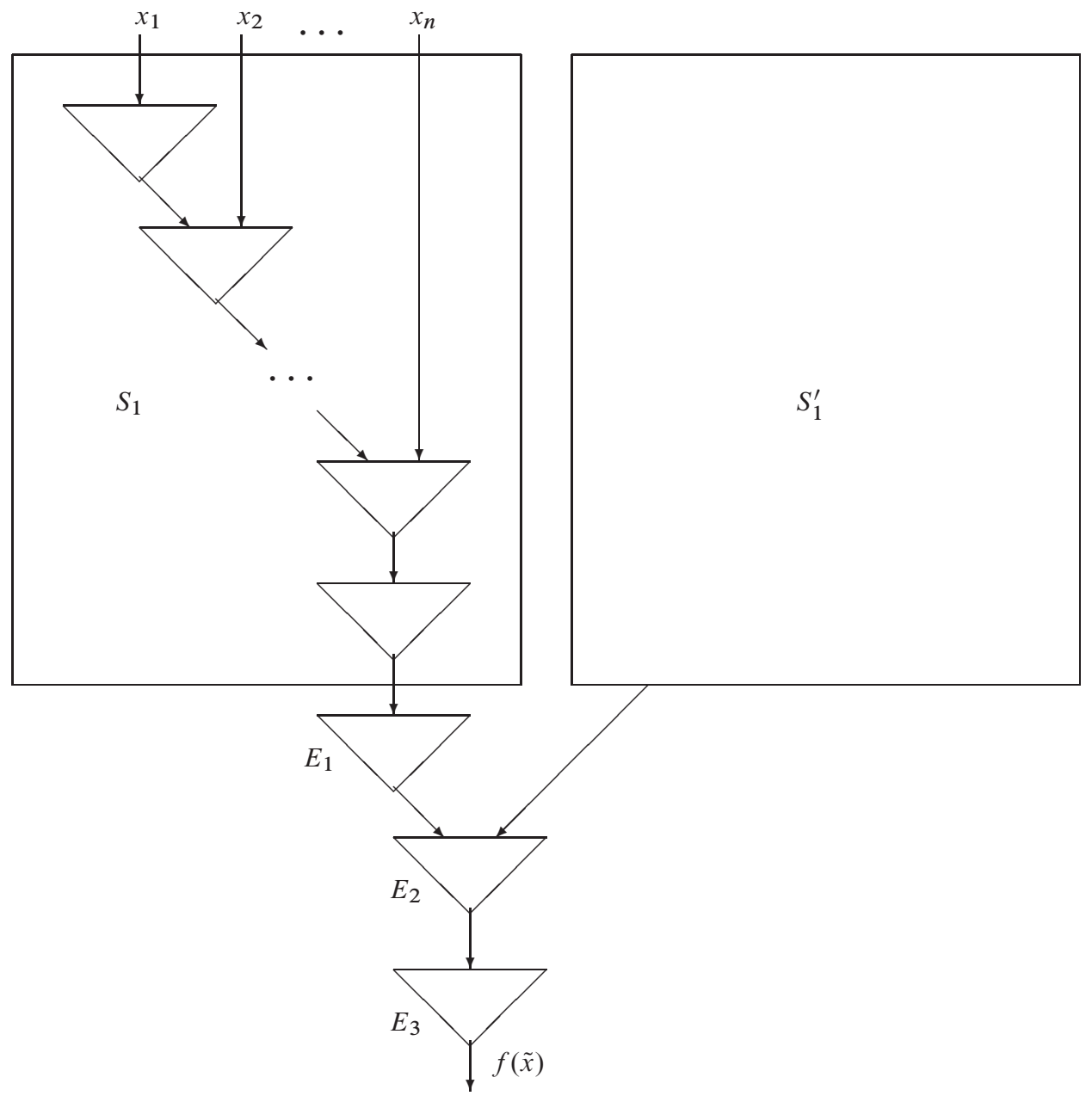

Рис. 5.

при неисправности инвертора $E_{1} ; h_{n+2}=\bar{f}$ при неисправности одного из элементов $E_{2}, E_{3}$.

Итак, на выходе схемы могут получиться всего две различные неисправности, следовательно, $D_{\text {Едт }} \leqslant 2$.

6. Базисы, содержащче функциию $x \vee y$. Для таких базисов требуемое утверждение доказано в работе [6] (теорема 6).

7. Базисы, содержащчие функциюю $x \& y$. Расширением базиса В будем считать (как и в [6]) всякий базис $B^{\prime}$, любая функция которого либо совпадает с какой-нибудь функцией из $B$, либо может быть получена путем отождествления переменных какой-нибудь функции из $B$. Любая схема, построенная в базисе $B^{\prime}$, является схемой и в базисе $B$. Следовательно, достаточно доказать теорему для базиса $B^{\prime}$. Пусть $B^{*}-$ максимальное (по числу содержащихся в нем попарно различных функций) расширение базиса $B$. Если $B^{*}$, кроме функции $x \& y$, содержит и функцию $\bar{x}$, то строим схему в базисе $\{x \& y, \bar{x}\}$ (см. случай 2). 


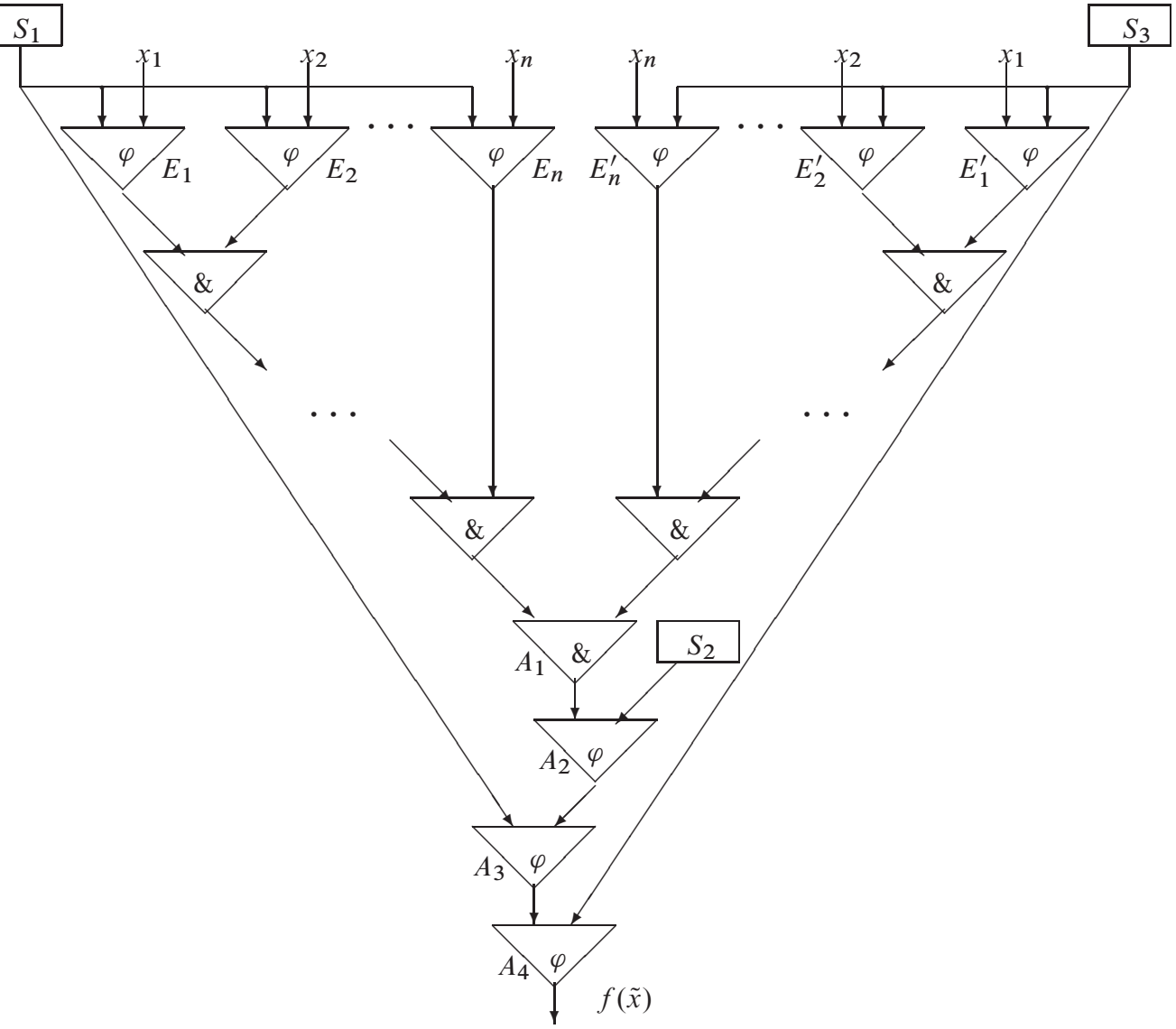

Pис. 6.

Пусть теперь $B^{*}$ не содержит $\bar{x}$. Базис $B$ содержит функцию, не сохраняющую константу 0 , из которой путем отождествления переменных можно получить либо инверсию, либо константу 1 , и функцию, не сохраняющую константу 1 , из которой аналогично можно получить либо инверсию, либо константу 0 . Но $B^{*}$ не содержит инверсию, значит, $B^{*}$ содержит обе константы. Базис $B$ (а значит, и $B^{*}$ ) содержит немонотонную функцию $\varphi$. По лемме о немонотонной функции [7], из нее можно получить инверсию путем подстановки вместо некоторых переменных констант.

Рассмотрим схему в базисе $B^{*}$, представленную на рис. 6. На этом рисунке каждая из подсхем $S_{1}, S_{2}, S_{3}$ содержат по два элемента, реализующих булевы константы (если для получения инверсий $\bar{x}_{1}, \ldots, \bar{x}_{n}$ можно ограничиться подачей на входы элементов верхнего яруса схемы, реализующих функцию $\varphi$, лишь какой-то одной булевой константы, то подсхемы $S_{1}, S_{2}, S_{3}$ содержат по одному элементу).

При наличии неисправного элемента в подсхеме $S_{1}$ элементы $E_{1}, \ldots, E_{n}, A_{3}$ будут выдавать либо константу 0 , либо константу 1 , либо тождественную функцию (относительно переменной $x$, подаваемой на правый вход элемента); при этом, как нетрудно заметить, на выходе всей схемы получим одну из булевых констант. Такие же функции неисправности возможны и при неисправности элемента в $S_{3}$. При неисправности элемента в $S_{2}$ на выходе всей схемы получим либо какую-нибудь булеву константу, либо $\bar{f}$. Наконец, 


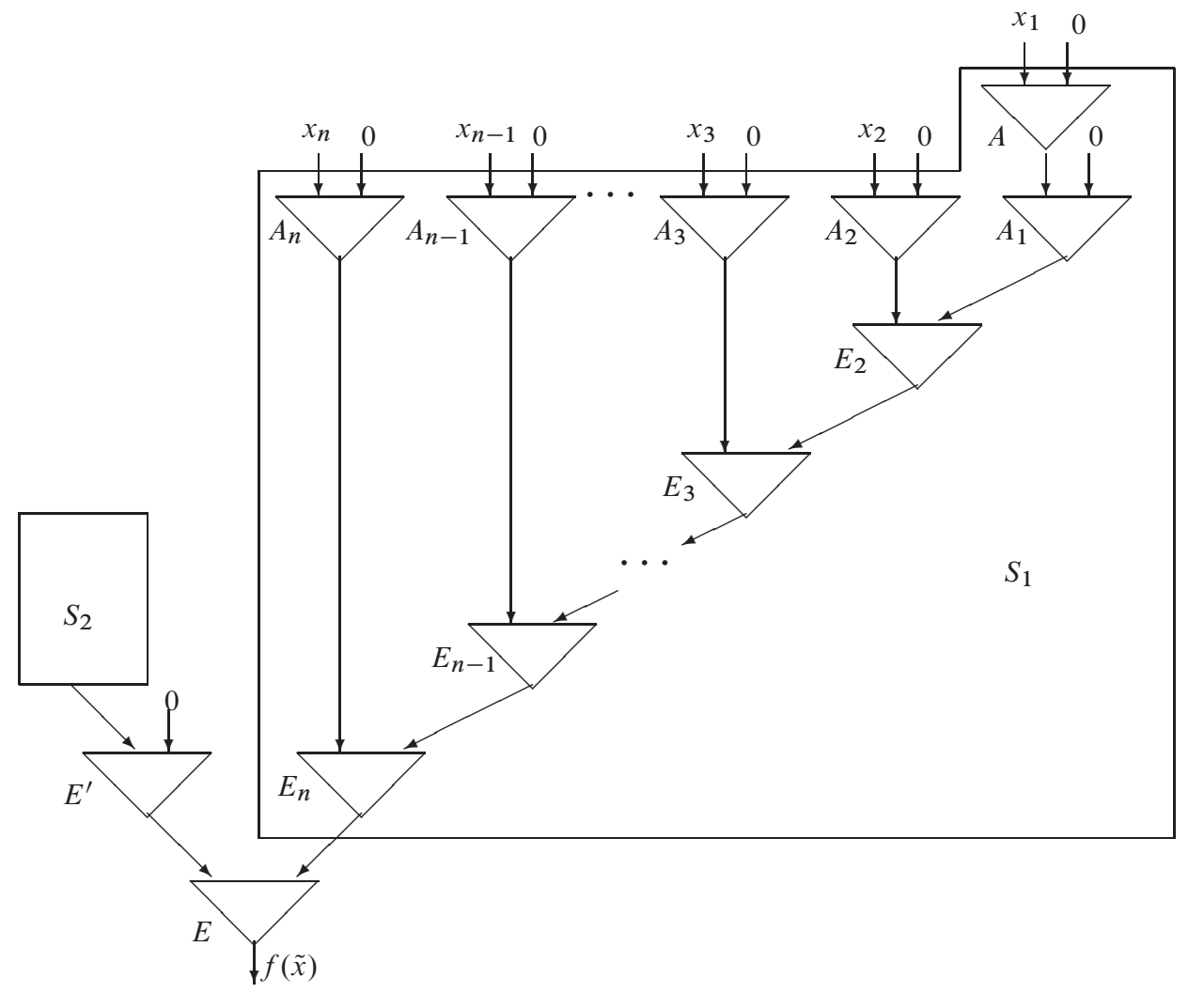

Рис. 7.

при неисправности одного из элементов $A_{1}, \ldots, A_{4}$ на выходе схемы получим $\bar{f}$, а при неисправности любого отличного от $A_{1}$ конъюнктора - константу 1 . Таким образом, всего можно получить не более трех различных функций неисправности (а именно, 0,1 и $\bar{f})$. Очевидно, что $\{\tilde{0}, \tilde{1}\}$ является единичным диагностическим тестом и утверждение теоремы выполняется.

8. Базисы, содержсащие функиию $\bar{x} \vee y$ или $\bar{x} \& y$. Если в $B^{*}$ содержится инверсия, то мы получим один из рассмотренных случаев 1 или 5 соответственно. Пусть теперь в $B^{*}$ нет инверсии, тогда в $B^{*}$ содержатся обе константы. Схема $S$ в базисе $\{\bar{x} \vee y, 0\}$ показана на рис. 7. Каждый двухвходовой элемент в $S$ реализует импликацию, а константа 0 , подаваемая на правые входы двухвходовых элементов, получается с использованием единственного во всей схеме элемента $E_{1}$, реализующего данную константу; подсхема $S_{2}$ устроена точно так же, как и $S_{1}$.

Выпишем функции $a, a_{1}, a_{2}, a_{3}, \ldots, a_{n}, e_{2}, e_{3}, \ldots, e_{n}$, которые реализуются на выходе подсхемы $S_{1}$ при переходе в неисправное состояние элементов $A, A_{1}, A_{2}, A_{3}, \ldots, A_{n}$, $E_{2}, E_{3}, \ldots, E_{n}$ соответственно:

$$
\begin{aligned}
a & =a_{1}=\bar{x}_{1} \vee x_{2} \vee \ldots \vee x_{n} ; \quad a_{2}=x_{1} \vee \bar{x}_{2} \vee x_{3} \vee \ldots \vee x_{n} ; \\
a_{3} & =x_{1} \vee x_{2} \vee \bar{x}_{3} \vee x_{4} \vee \ldots \vee x_{n} ; \ldots ; a_{n}=x_{1} \vee \ldots \vee x_{n-1} \vee \bar{x}_{n} ; \\
e_{2} & =\overline{x_{1} \vee x_{2} \vee x_{3} \vee \ldots \vee x_{n} ;} \\
e_{3} & =\overline{x_{1} \vee x_{2} \vee x_{3}} \vee x_{4} \vee \ldots \vee x_{n} ; \ldots ; e_{n}=\overline{x_{1} \vee \ldots \vee x_{n}}
\end{aligned}
$$


Отсюда следует, что при наличии неисправного элемента в подсхеме $S_{1}$ на выходе всей схемы будет реализована константа 1; эта же самая функция неисправности будет реализована и при наличии неисправного элемента в подсхеме $S_{2}$. Легко заметить также, что на выходе схемы $S$ получим $\bar{f}$ при неисправности выходного элемента $E$ или константу 1 при неисправности одного из элементов $E^{\prime}, E_{1}$ (напомним, что в исправном состоянии $E_{1}$ реализует константу 0 ). Всего для схемы $S$ возможны две разные нетривиальные функции неисправности и утверждение теоремы для нее справедливо.

Для базиса $\{\bar{x} \& y, 1\}$ утверждение теоремы доказывается аналогичным образом с использованием схемы $S^{*}$, которая представлена на рис. 8. Нетрудно убедиться, что для схемы $S^{*}$ возможны только две нетривиальные функции неисправности ( $\bar{f}$ и константа 1$)$ и утверждение теоремы для $S^{*}$ выполняется.

9. Базисы, содержащие функиию

$$
\varphi=x y \oplus x z \oplus y z \oplus \alpha_{1} x \oplus \alpha_{2} y \oplus \alpha_{3} z \oplus \alpha_{4} .
$$

Для базиса $B^{*}$ в [8] показано, что если в $B^{*}$ отсутствуют константы, то $B^{*}$ содержит по крайней мере одну из функций $x^{\sigma} y^{\sigma}, x^{\sigma} \vee y^{\sigma}(\sigma \in\{0,1\})$; базисы, содержащие такие функции, уже рассмотрены. Далее считаем, что $B^{*}$ содержит по крайней мере одну булеву константу $\beta$. Если константа $\bar{\beta}$ отсутствует в $B^{*}$, то возьмем подсхему (блок) $E^{*}$ из двух элементов $E^{\prime}, E^{\prime \prime}$, где $E^{\prime}$ реализует константу $\beta, E^{\prime \prime}$ реализует функцию, не сохраняющую константу $\beta$ (такая функция в силу полноты исходного базиса обязательно присутствует в $\left.B^{*}\right)$, и все входы элемента $E^{\prime \prime}$ соединены с выходом элемента $E^{\prime}$, а выход элемента $E^{\prime \prime}$ является выходом блока $E^{*}$. Исправный блок $E^{*}$ реализует константу $\bar{\beta}$, а единственной нетривиальной функцией неисправности этого блока является константа $\beta$. Следовательно, данный блок можно рассматривать как функциональный элемент, реализующий константу $\bar{\beta}$, и полагать далее, что базис содержит элементы, реализующие обе булевы константы. В зависимости от значений $\alpha_{1}, \alpha_{2}$ и $\alpha_{3}$ достаточно рассмотреть четыре варианта функции $\varphi$ :

$$
\begin{aligned}
& \varphi_{1}=x y \oplus x z \oplus y z \oplus x \oplus y \oplus z \oplus \alpha_{4} ; \\
& \varphi_{2}=x y \oplus x z \oplus y z \oplus x \oplus y \oplus \alpha_{4} ; \\
& \varphi_{3}=x y \oplus x z \oplus y z \oplus x \oplus \alpha_{4} ; \\
& \varphi_{4}=x y \oplus x z \oplus y z \oplus \alpha_{4} ; \quad \alpha_{4} \in\{0,1\} ;
\end{aligned}
$$

все остальные варианты сводятся к указанным переименованием переменных. Рассмотрим первый вариант. Ясно, что

$$
\varphi_{1}=x y \oplus x z \oplus y z \oplus x \oplus y \oplus z \oplus \alpha_{4} \in B^{*}
$$

и $\varphi_{1}$ реализуется на выходе трехвходового элемента $E$. При подстановке в $\varphi_{1}$ константы 0 вместо $z$ получаем функцию

$$
\varphi_{1}(x, y)=x y \oplus x \oplus y \oplus \alpha_{4}=(x \vee y) \oplus \alpha_{4} ;
$$

эта функция реализуется на выходе элемента $E$ при подаче на первые два входа этого элемента переменных $x, y$ и константы 0 (вместо $z$ ) на третий вход.

Предположим, что $\alpha_{4}=0$. Возьмем схему $S$, представленную в [6] на рис. 1 (в схеме на входы выходного дизъюнктора подаются выходы двух одинаковых цепочек из дизъюнкторов длины $n$, каждая из которых реализует $x_{1} \vee \ldots \vee x_{n}$ ); в исправном состоянии $S$ 


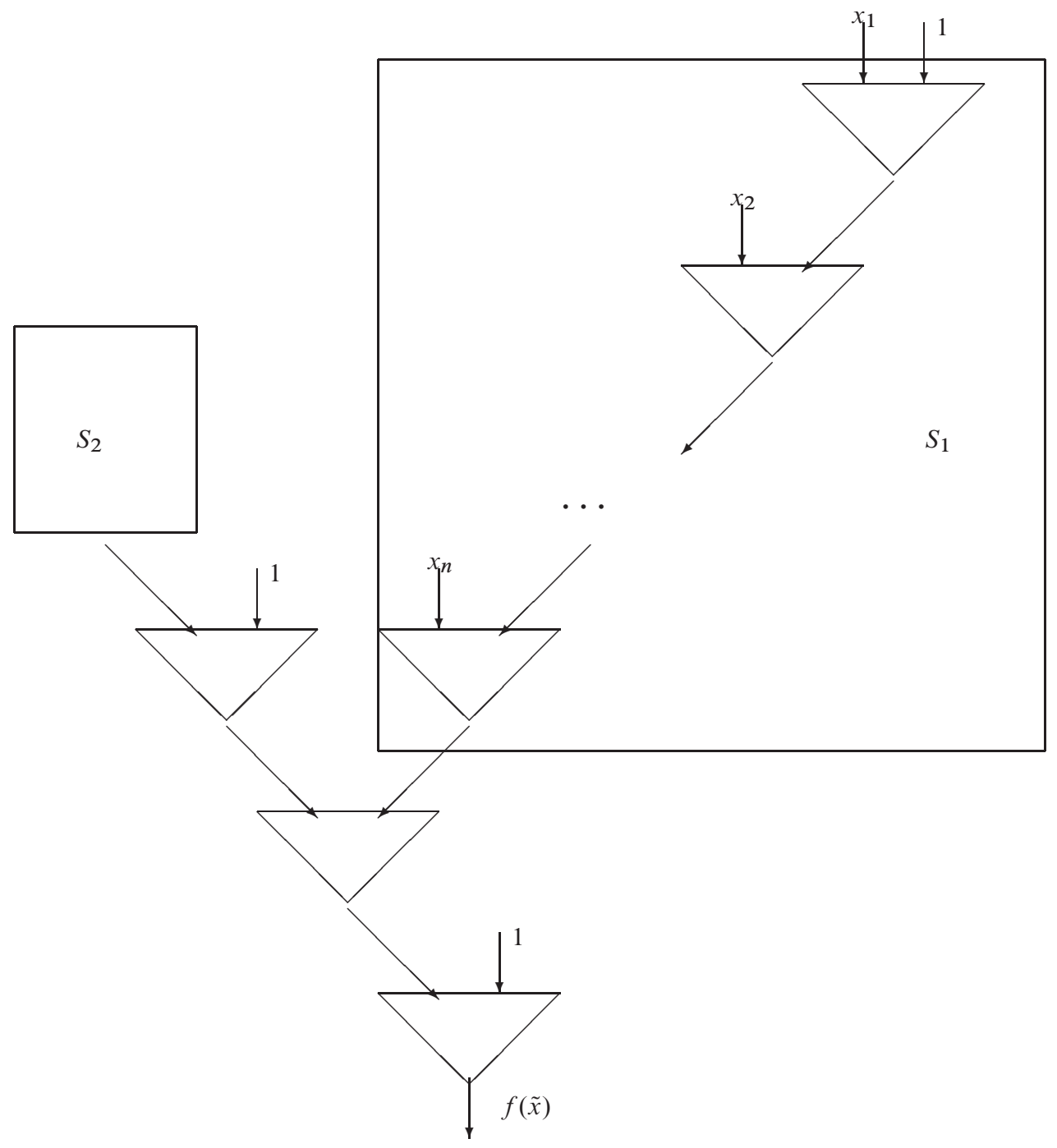

Рис. 8.

реализует $x_{1} \vee \ldots \vee x_{n}$, а в неисправных состояниях - нетривиальные функции неисправности $g_{1} \equiv 1$ и $g_{2}=\overline{x_{1} \vee \ldots \vee x_{n}}$. В $S$ добавим $2 n+1$ элемент, реализующие константу 0 ; все дизъюнкторы заменим на трехвходовые элементы $E$, соединив третий вход каждого элемента $E$ с выходом соответствующего ему элемента $E_{0}$, и получим некоторую схему $S^{\prime}$, реализующую, как и $S$, дизъюнкцию $x_{1} \vee \ldots \vee x_{n}$. При переходе одного из элементов $E_{0}$ в неисправное состояние, на выходе $S^{\prime}$ будет реализована одна из следующих функций неисправности: $\bar{x}_{1} \vee \bar{x}_{2} \vee x_{3} \vee \ldots \vee x_{n} \vee f \equiv 1, \ldots, \overline{x_{1} \vee \ldots \vee x_{n-1}} \vee \bar{x}_{n} \vee f \equiv 1, \bar{f} \vee \bar{f}=\bar{f}$. При переходе в неисправное состояние любого одного элемента $E$ в схеме $S^{\prime}$ на выходе этой схемы будет реализована (как и на выходе исходной схемы $S$ ) одна из нетривиальных функций неисправности $1, \bar{f}$. В итоге схеме $S^{\prime}$ отвечают две нетривиальные функции неисправности и $D_{\text {Едт }} \leqslant 2$.

Если $\alpha_{4}=1$, то в качестве исходной берем схему в базисе $\{\bar{x} \& \bar{y}\}$, представленную 
на рис. 2, и, фактически повторяя вышеизложенные рассуждения (для случая $\alpha_{4}=0$ ), убеждаемся, что и при $\alpha_{4}=1$ можно построить схему $S^{\prime}$, для которой $D_{\text {Едт }}\left(S^{\prime}\right) \leqslant 2$.

Остальные три варианта рассматриваются аналогично. Для $\varphi_{2}$ рассуждения те же, что и для $\varphi_{1}$. Для $\varphi_{3}$ полагаем $x \equiv 1$ и далее проводим рассуждения, как для $\varphi_{1}$. Для $\varphi_{4}$ полагаем $z \equiv 1$ и далее рассуждения опять же, что и для $\varphi_{1}$. В каждом случае утверждение теоремы выполняется.

10. Произвольный базис. Пусть $B$ - произвольный полный конечный базис. В [6] показано, что максимальное расширение $B^{*}$ базиса $B$ содержит либо одну из функций $x^{\alpha} y^{\beta}, x^{\alpha} \vee y^{\beta}$, где $\alpha, \beta \in\{0,1\}$, либо функцию

$$
\varphi=x y \oplus x z \oplus y z \oplus \alpha_{1} x \oplus \alpha_{2} y \oplus \alpha_{3} z \oplus \alpha_{4},
$$

где $\alpha_{1}, \ldots, \alpha_{4} \in\{0,1\} ;$ соответствующие базисы уже рассмотрены.

Теорема доказана.

Предложение 1. Приведенная в теореме оценка в общем случае неулучшаема.

Доказательство. В [6] это утверждение было доказано для схем без ветвлений выходов элементов, то есть для формул.

Заметим, что любая схема для функции $f(\tilde{x})$ в случае неисправности выходного элемента реализует нетривиальную функцию неисправности $g_{1}(\tilde{x})=\bar{f}(\tilde{x})$. С учетом этого обстоятельства для доказательства предложения возьмем базис $\{x \vee y, \bar{x}\}$ и покажем, что для любой неизбыточной схемы $S$ в данном базисе, реализующей $f(\tilde{x})=x_{1} \vee \ldots \vee x_{n}$, возможна неисправность, при которой на выходе схемы $S$ получается нетривиальная функция неисправности $g_{2}(\tilde{x})$, отличная от $g_{1}(\tilde{x})$.

В схеме $S$, реализующей $f(\tilde{x})$, выделим цепь $Z$ из элементов, удовлетворяющую следующим условиям:

(a) начинается $Z$ (верхним) двухвходовым элементом $E_{1}$, то есть дизьюнктором;

(б) заканчивается $Z$ (нижним) элементом $E_{k}$, являющимся выходным элементом схемы $S$;

(в) входы верхнего элемента $E_{1}$ соединены с различными вершинами $V_{1}$ и $V_{2}$ схемы $S$ и хотя бы одна из этих вершин, например, $V_{1}$, является выходом некоторого элемента $E^{\prime}$ схемы $S$;

(г) каждый вход элемента $E_{i}$ из $Z, i=2,3, \ldots, k$, соединен с выходом элемента $E_{i-1}$ в случае, когда $E_{i}$ инвертор, и оба входа элемента $E_{i}$ соединены с выходами элемента $E_{i-1}$ в случае, когда $E_{i}$ дизъюнктор (см. рис. 9);

(д) цепь $Z$ имеет наибольшую возможную длину при указанных условиях.

Учитывая, что $S$ реализует дизъюнкцию не менее чем трех переменных, легко заметить, что указанная непустая цепь $Z$ существует и находится единственным образом. Очевидно также, что на выходе элемента $E_{1}$ реализуется либо $f(\tilde{x})$, либо $\bar{f}(\tilde{x})$.

Предположим, что в цепи $Z$ содержится нечетное число инверторов, а на выходе элемента $E_{1}$ реализуется $\bar{f}(\tilde{x})=\overline{x_{1} \vee \ldots \vee x_{n}}$. В этом случае либо в каждой из вершин $V_{1}, V_{2}$ реализуется $\bar{f}$, либо в одной из вершин реализуется константа 0 , а в другой $\bar{f}$. Если в вершине $V_{i}$ реализуется константа 0 , то $V_{i}$ - выход элемента, и при неисправности этого элемента на его выходе будет реализована константа 1 , а на выходе всей схемы константа 0 ; ясно, что в этом случае $g_{2}(\tilde{x}) \not \equiv g_{1}(\tilde{x})$. 


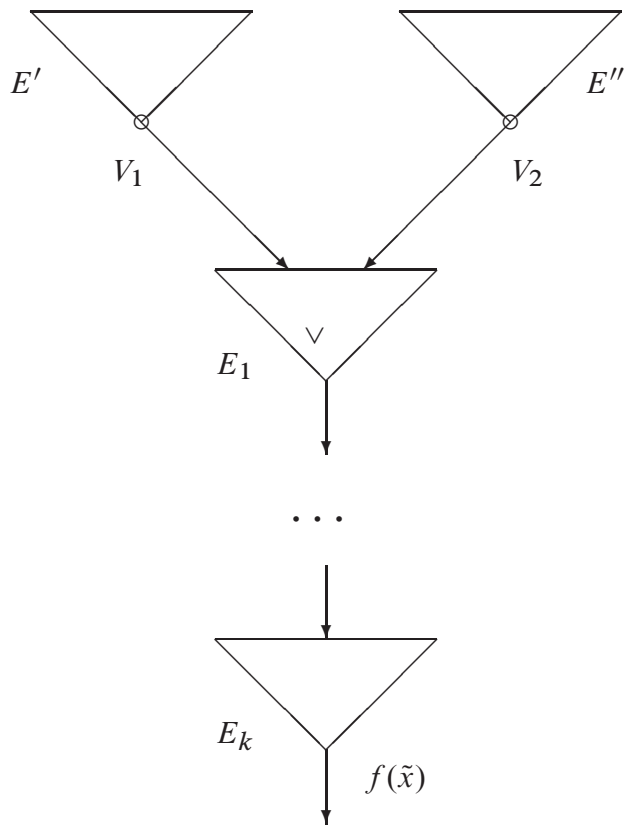

Рис. 9.

Пусть в вершинах $V_{1}, V_{2}$ реализуется одна и та же функция $\bar{f}$. Поскольку в схеме $S$ нет циклов, функция, реализуемая на выходе хотя бы одного из элементов $E^{\prime}, E^{\prime \prime}$, не зависит от состояния другого элемента; предположим, что на выходах элементов $E^{\prime}, E^{\prime \prime}$ реализуются соответственно функции $\varphi, \psi$ и функция $\psi$ не зависит от состояния элемента $E^{\prime}$. При неисправности элемента $E^{\prime}$ на его выходе, а вслед за ним и на выходе всей схемы опять же будет реализована константа и опять же получаем, что $g_{2}(\tilde{x}) \not \equiv g_{1}(\tilde{x})$.

Далее будем считать, что в цепи $Z$ содержится четное число инверторов, а это означает, что на выходе элемента $E_{1}$ реализуется $f$. Рассмотрим отдельно два случая.

1. $V_{1}, V_{2}$ - выходы двух разных элементов $E^{\prime}$ и $E^{\prime \prime}$, реализующих $\varphi$ и $\psi$ соответственно.

2. $V_{1}$ - выход элемента $E^{\prime}$, а $V_{2}$ - вход схемы, отвечающий, скажем, переменной $x_{1}$.

Рассмотрим первый случай. Как было замечено выше, без ограничения общности можно предположить, что функция $\psi$ не зависит от состояния элемента $E^{\prime}$. Если $\varphi \equiv \psi$, то при неисправности элемента $E^{\prime}$ на выходе элемента $E_{1}$, а значит, и на выходе всей схемы будет реализована булева константа; получаем отличную от $\bar{f}$ функцию неисправности $g_{2}$.

Пусть $\varphi \not \equiv \psi$. Если $\psi \equiv 0$, то при неисправности элемента $E^{\prime \prime}$ на выходе схемы получим константу и $g_{2} \not \equiv g_{1}$. Будем считать ниже, что $\psi \not \equiv 0$; заметим также, что $\varphi(\tilde{0})=\psi(\tilde{0})=0$, иначе на выходе исправной схемы $S$ для входного набора $\tilde{0}$ получим 1 , а не 0 . Поскольку $\psi$ не является константой 0 , найдется набор $\tilde{\sigma}$, отличный от $\tilde{0}$, на котором $\psi(\tilde{\sigma})=1$. При неисправности элемента $E^{\prime}$ для функции неисправности $g_{2}$ будет выполнено равенство $g_{2}(\tilde{0})=g_{2}(\tilde{\sigma})$, а это означает, что $g_{2} \not \equiv g_{1}$.

Во втором случае при неисправности элемента $E^{\prime}$ для $g_{2}$ выполняется условие $g_{2}(\tilde{0})=g_{2}(\tilde{1})$, свидетельствующее о том, что $g_{2} \not \equiv g_{1}$.

При наличии двух попарно различных нетривиальных функций неисправности предложение следует, например, из теоремы 21 в [2]. 
Автор выражает огромную благодарность своему научному руководителю Н. П. Редькину за постановку задачи и внимание к работе.

\section{Список литературы}

1. Лупанов О. Б., Асимптотические оценки сложности управляющих систем. Изд-во МГУ, Москва, 1984.

2. Редькин Н. П., Дискретная математика. Изд-во ЦПИ при мех.-мат. ф-те МГУ, Москва, 2007.

3. Редькин Н. П., Надежность и диагностика схем. Изд-во МГУ, Москва, 1992.

4. Яблонский С. В., Некоторые вопросы надежности и контроля управляющих систем. Математические вопросы кибернетики (1988) 1, 5-25.

5. Редькин Н. П., Единичные проверяющие тесты для схем при инверсных неисправностях элементов. Математические вопросы кибернетики (2003) 12, 217-230.

6. Беджанова С. Р., О минимальных тестах для схем, реализующих дизъюнкцию. Дискретный анализ и исследование операщий (2008) 15, №2, 3-11.

7. Яблонский С. В., Введение в дискретную математику. Наука, Москва, 1986.

8. Редькин Н. П., О полных проверяющих тестах для схем из функциональных элементов. Математические вопросы кибернетики (1992) 2, 198-222.

Статья поступила 15.04.2010. 